This is a postprint version of the following published document:

Lo, M.-C., Guzmán, R. Ali, M., Santos, R., Augustin, L. \& Carpintero, G. (2017). Sub-picosecond pulse and terahertz optical frequency comb generation by monolithically integrated linear mode-locked laser. Proceedings SPIE 10242, Integrated Optics: Physics and Simulations III (May 17, 2017), 102420A.

DOI: $10.1117 / 12.2264930$

(C) SPIE, 2017 


\title{
Sub-picosecond pulse and terahertz optical frequency comb generation by monolithically integrated linear mode-locked laser
}

\author{
Mu-Chieh Lo ${ }^{\mathrm{a}}$, Robinson Guzmán ${ }^{\mathrm{a}}$, Muhsin $\mathrm{Ali}^{\mathrm{a}}$, Rui Santos ${ }^{\mathrm{b}}$, Luc Augustin ${ }^{\mathrm{b}}$, Guillermo Carpintero ${ }^{\mathrm{a}}$ \\ ${ }^{a}$ Universidad Carlos III de Madrid, Av. Universidad 30, 28911 Leganés, Spain \\ ${ }^{\mathrm{b}}$ SMART Photonics, Horsten 1, 5612 AX Eindhoven, the Netherlands The Netherlands
}

\begin{abstract}
We report on a record broad 3-dB bandwidth of $14 \mathrm{~nm}(\sim 1.8 \mathrm{THz}$ around $1532 \mathrm{~nm})$ optical frequency comb generated from a passively mode-locked quantum-well (QW) laser in the form of photonic integrated circuits through an InP generic photonic integration technology platform. This 21.5-GHz colliding-pulse mode-locked laser cavity is defined by two on-chip reflectors incorporating intracavity phase modulators followed by an out-of-cavity SOA as booster. Under certain operating conditions, an ultra-wide spectral bandwidth is achieved along with an autocorrelation trace confirming the mode locking nature exhibiting a pulse width of $0.35 \mathrm{ps}$. The beat note RF spectrum has a linewidth of sub-MHz and 35-dB SNR.
\end{abstract}

Keywords: Photonic integrated circuits, Mode-locked lasers, Optical frequency comb generation, Ultrafast pulse

\section{INTRODUCTION}

Integrated passively mode-locked semiconductor lasers are robust and compact sources for generating coherent optical frequency combs which are of great interests in communications, metrology, spectroscopy and millimeter wave/terahertz $(\mathrm{mmW} / \mathrm{THz})$ generation [1-3]. Unlike other optical frequency comb generation techniques, it does not require any external RF source or optical pump.

A passively mode-locked laser is usually a two-section structure, composed of a semiconductor optical amplifier (SOA) as gain section and a saturable absorber (SA) in a Fabry-Pérot cavity [4-6]. Alternatively, a passively mode-locked laser can be designed in such a way that SAs are placed at some certain positions within the resonator to support two or more pulses meeting in the absorbers that is the colliding-pulse mode locking scheme [7-10].

When developed in active-passive integration, passive waveguides and components, namely multimode interference couplers/reflectors (MMIs/MIRs), electro-optic phase modulators (EOPMs) are enabled to bring in more functionalities and design freedom to the photonic integrated circuits (PICs) [11]. As the SOA and SA lengths play an important role in determining the performance quality of mode-locked laser, the use of passive waveguides reduces the self-phase modulation in active medium [12,13]. Using MMIs and curved waveguides, mode-locked lasers in ring geometry are feasible [14,15]. MIRs allow the on-chip Fabry-Pérot cavity [16], signal acquisition and post-stage processing [17]. In [18-20] it was shown that by introducing EOPM-based intracavity gain flattening filter, the optical bandwidth, which is the key characteristic of a comb, can be significantly improved.

Recent studies on the bandwidth of comb generated from a passively mode-locked semiconductor laser have shown that the performance of quantum-dash (Q-dash) and quantum-dot (QD) active regions is superior to that of quantum-well (QW) [21,22]. A QD-based passively mode-locked laser with a 12-nm 3-dB optical bandwidth was presented in [23]. A frequency comb of more than $16 \mathrm{~nm}$ was demonstrated from a Q-dash laser in [24]. In [25] a 3-dB optical bandwidth of $11.5 \mathrm{~nm}$ was reached with a $20-\mathrm{GHz}$ ring passively mode-locked laser, which was a record value for QW. In [26], the same group proposed another design using the same technology to generate a wide comb with 42-nm 20-dB bandwidth.

In this paper, we present an integrated mode-locked quantum-well laser developed through a generic InP photonic integration platform with a new record bandwidth of $14 \mathrm{~nm}$ that is comparably wider than passively mode-locked QDbased laser. The proposed design is a colliding-pulse mode-locked laser, comprising a saturable absorber (SA), gain sections (SOA), passive waveguide and multimode interference reflectors (MIR) in a symmetric arrangement. New features that we have included in this device are symmetrically positioned intracavity phase modulators (EOPM) and out-of-cavity SOA. Using MIR mirrors allows to access the optical outputs on chip, enabling further functionalities on 
the signal, as amplification though the out-of-cavity SOA. The device is demonstrated to generate an extremely broad optical spectrum, that constitutes an optical frequency comb. The phase locking of modes is agreed with the autocorrelation trace, showing ultrafast pulses. The optical comb has a record 3-dB bandwidth of $14 \mathrm{~nm}(1.8 \mathrm{THz})$, even wider than that of QD-based passively mode-locked laser.

\section{DEVICE DESCRIPTION AND EXPERIMENTAL SETUP}

Fig. 1(a) depicts the proposed PIC layout and Fig. 1(b) presents the dimension table. This PIC was developed through a generic approach and fabricated within a multi-project wafer (MPW) run by SMART Photonics [27]. The process design kit (PDK) provides a selection of predefined optoelectronic components as parametric building blocks [28]. The PIC presented in Fig. 1(a) is composed of a saturable absorbor (SA), semiconductor optical amplifiers (SOA), electro-optic phase modulators (EOPM), multimode interference reflectors (MIR) and straight/bent passive waveguides in a symmetric geometry with respect to the SA. This symmetry configuration assures operation in colliding-pulse mode locking regime [29], and the total cavity length defined by MIRs is $3.78 \mathrm{~mm}$ corresponding to a repetition rate of about $21.5 \mathrm{GHz}$. This $30-\mu \mathrm{m} \mathrm{SA}$ is surround by two $400-\mu \mathrm{m}$ SOAs with two $1200-\mu \mathrm{m}-$ long EOPMs on both sides. Between active components isolations were placed to avoid unwanted current flows. Transitions were inserted between deeply etched and shallowly etched waveguides. The two MIRs have 50\% reflectivity and 50\% transmissivity on two ports. In both MIRs, one port connects to the active components and form a linear resonant cavity. The other port either sends out the pulse train directly to the facet edge through the left $7^{\circ}$ angled waveguide (OUT1) or to the other $7^{\circ}$ angled waveguide (OUT2) via the 400- $\mu \mathrm{m}$ booster amplifier (SOA3). The deeply etched waveguides have a width of $1.5 \mu \mathrm{m}$ and a radius of $100 \mu \mathrm{m}$. A metal pad for electrical contact was placed and routed to each active component.

(a)

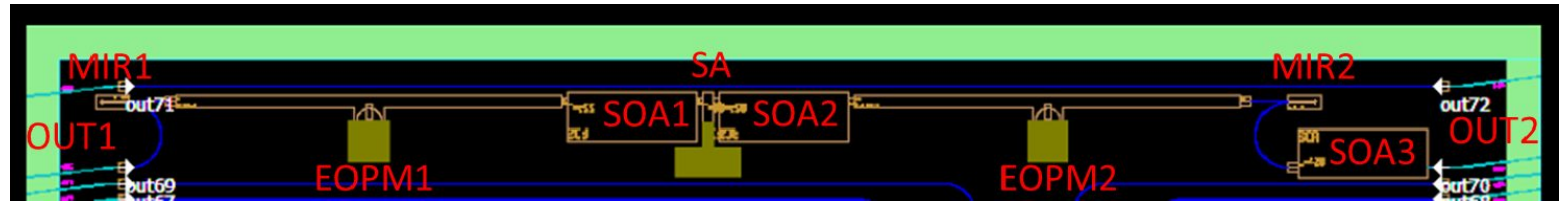

(b)

\begin{tabular}{|l|l|l|l|l|l|l|}
\hline SA & SOA1 & SOA2 & SOA3 & EOPM1 & EOPM2 & Cavity \\
\hline $30 \mu \mathrm{m}$ & $400 \mu \mathrm{m}$ & $400 \mu \mathrm{m}$ & $400 \mu \mathrm{m}$ & $1200 \mu \mathrm{m}$ & $1200 \mu \mathrm{m}$ & $3780 \mu \mathrm{m}$ \\
\hline
\end{tabular}

Figure 1. (a) Schematic layout. (b) Dimension table. SA: saturable absorber. SOA: semiconductor optical amplifier. EOPM: electro-optic phase modulator.

For electrically driving the PIC, the metal pads of PIC were wire-bonded to printed circuit board as the photo shown in Fig. 2. A block diagram of experimental setup is presented where the PIC were biased with voltage sources (Agilent E3634A Power Supplies) and current sources (Thorlabs PRO8000 LD Controller), denoted as Veopm1,2 (for EOPM1 and EOPM2), Vsa, and Isoa1,2,3 (for SOA1, SOA2, and SOA3), respectively. The PIC was mounted on a copper chuck controlled at $16^{\circ} \mathrm{C}$ with Thorlabs PRO8000 TEC Controller. The optical output signal from OUT2 was coupled to an Oz Optics lensed fiber. The collected optical signal was diagnosed on power meter (PM), Yokogawa AQ6370B optical spectrum analyzer (OSA), APE PulseCheck autocorrelator (AC) and Anritsu MS2668C electrical spectrum analyzer (ESA). We used Amonics EDFA (erbium-doped fiber amplifier) to pump the power up to $10 \mathrm{dBm}$ and a polarization controller (PC) prior to the autocorrelator. For the electrical beat tone measurement, Nortel EDFA with $10 \mathrm{dBm}$ output power and $\mathrm{u}^{2} \mathrm{t}$ photodiode (PD) with $40 \mathrm{GHz}$ bandwidth were employed. 


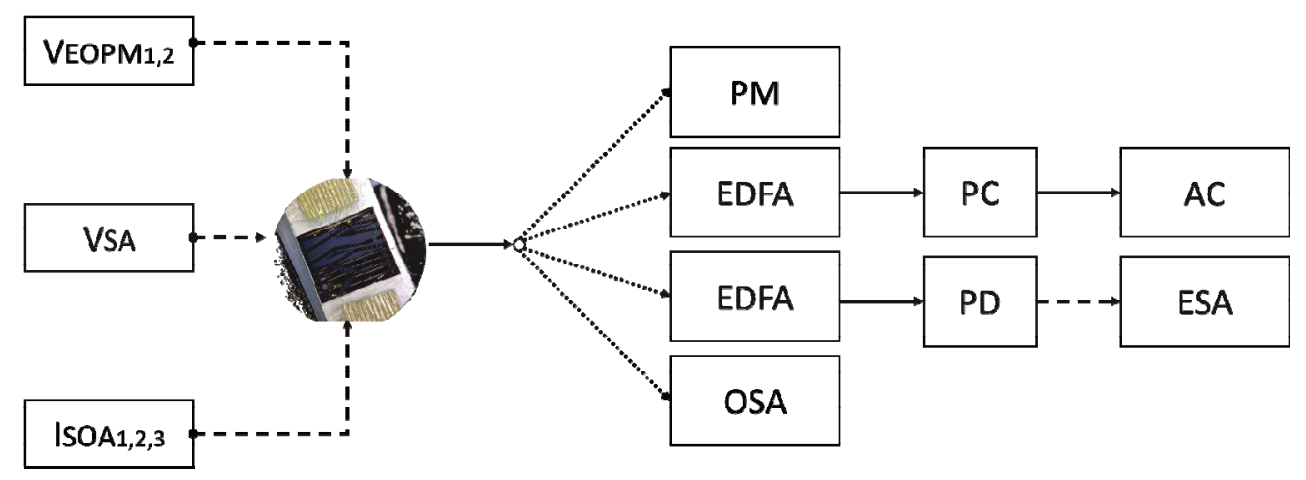

Figure 2. Experimental setup. PM: power meter. EDFA: erbium-doped fiber amplifier. PC: polarization controller. AC: autocorrelator. PD: photodetector. ESA: electrical spectrum analyzer. OSA: optical spectrum analyzer.

\section{RESULTS AND DISCUSSION}

The frequency comb generated from the PIC is demonstrated in Fig. 3 with a resolution bandwidth of $0.02 \mathrm{~nm}$. It exhibits an extremely broad spectrum with maximum level at $1537.44 \mathrm{~nm},-32 \mathrm{dBm} / \mathrm{res}$ and the spectral width at $3-\mathrm{dB}$ level is $14 \mathrm{~nm}$. Within the $3-\mathrm{dB}$ bandwidth, it covers 83 comb teeth and the mode spacing is about $0.172 \mathrm{~nm}$ corresponding to $21.5 \mathrm{GHz}$. However, the profile of spectrum is neither perfectly smooth nor symmetric. Three plateaus can be observed at around $1525.7 \mathrm{~nm}, 1530.2 \mathrm{~nm}$ and $1535.8 \mathrm{~nm}$. Also, the side mode suppression ratio is $8 \mathrm{~dB}$ which is relatively weak as the highest suppressed modes are approximately at $-40 \mathrm{dBm} / \mathrm{res}$. Further investigation on the spectrum shape improvement and optimized operating condition are required. The wide bandwidth leads to a very short temporal trace, as will be demonstrated in the next section.

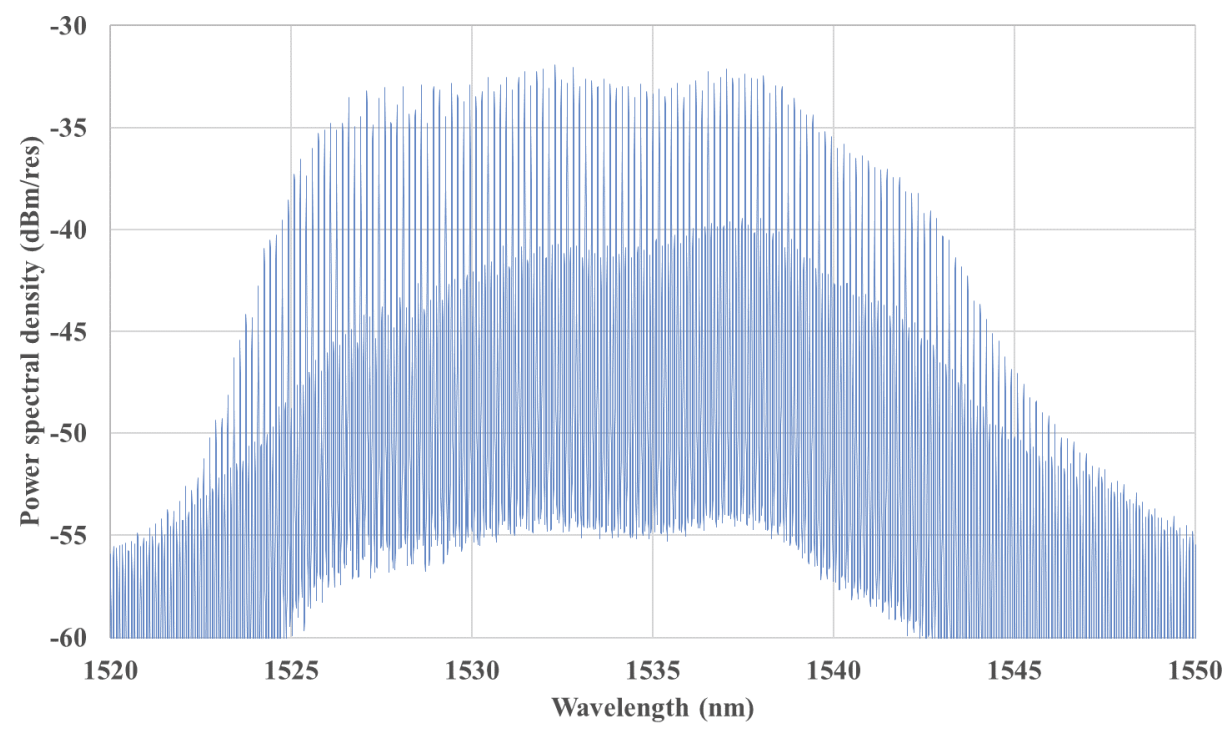

Figure 3. Optical spectrum when $\mathrm{Vsa}=-0.6 \mathrm{~V}$, Isoa1 $=$ Isoa2 $=80 \mathrm{~mA}$, Isoa3 $=30 \mathrm{~mA}$. Resolution bandwidth: $0.02 \mathrm{~nm} .3-$ $\mathrm{dB}$ bandwidth $=14 \mathrm{~nm}(1.8 \mathrm{THz})$. Side mode suppression ratio (SMSR) $\sim 8 \mathrm{~dB}$

In Fig. 4, at the same operating condition the autocorrelation (AC) trace is shown where a pulse appears at the midpoint of time delay span. The full width at half maximum (FWHM) of AC trace is $0.61 \mathrm{ps}$. The FWHM of pulse assuming a sech2 shape is 0.35 ps. Please note the pulse is standing on a pedestal. It may be attributed to the complicated intensities with slowly varying envelopes and the non-zero DC component amplified by SOA3 [30,31]. 


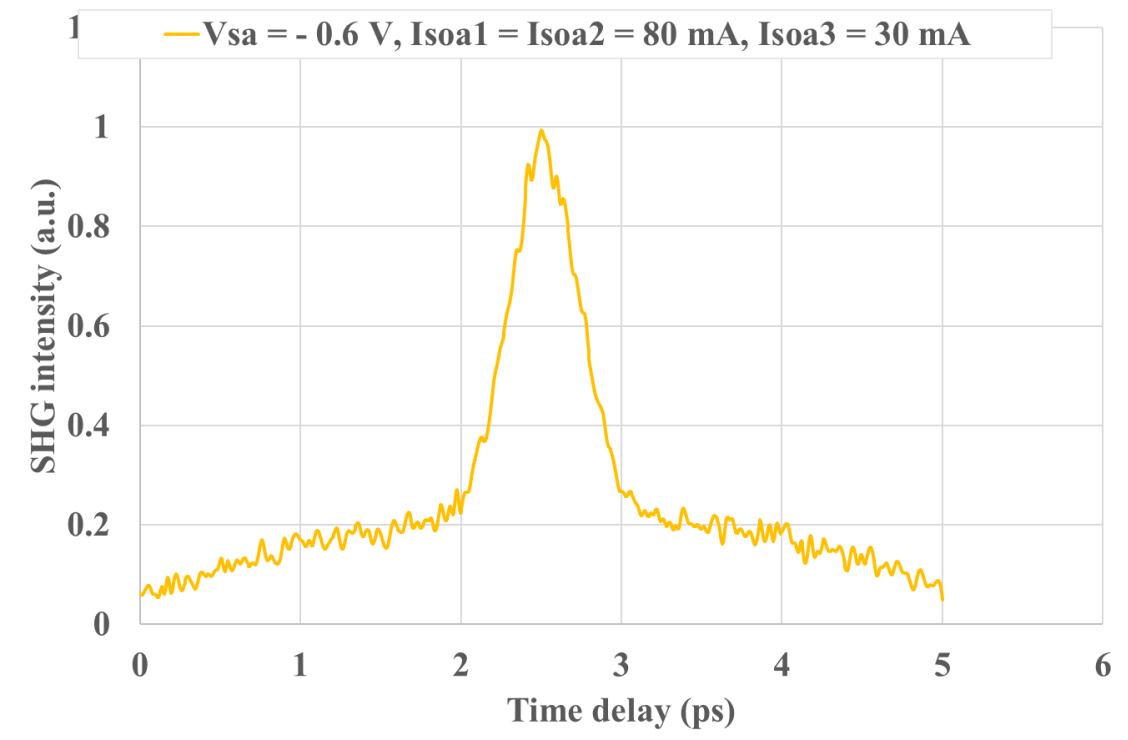

Figure 4. Autocorrelation trace when $\mathrm{Vsa}=-0.6 \mathrm{~V}$, Isoa1 $=\mathrm{Isoa} 2=80 \mathrm{~mA}$, Isoa3 $=30 \mathrm{~mA}$. Full width at half maximum of $\mathrm{AC}$ trace $\left(\mathrm{FWHM}_{\mathrm{AC}}\right)=0.61 \mathrm{ps}$. Assuming a sech 2 pulse shape $\mathrm{FWHM}_{\mathrm{Sech} 2}=0.35 \mathrm{ps}$.

The RF spectrum of beat note through the $40 \mathrm{GHz}$ photodiode is demonstrated in Fig. 5. Fig. 5(a) shows the full span up to $40 \mathrm{GHz}$ with $1-\mathrm{MHz}$ resolution bandwidth, while Fig. 5(b) shows the RF tone in detail with 3-kHz resolution bandwidth. In Fig. 5(a), the RF tone signal is at $21.5 \mathrm{GHz}$ and the signal noise ratio is about $35 \mathrm{~dB}$. In Fig. 5(b), the RF tone is observed at $21.512 \mathrm{GHz}$ with a linewidth of under $1 \mathrm{MHz}$.

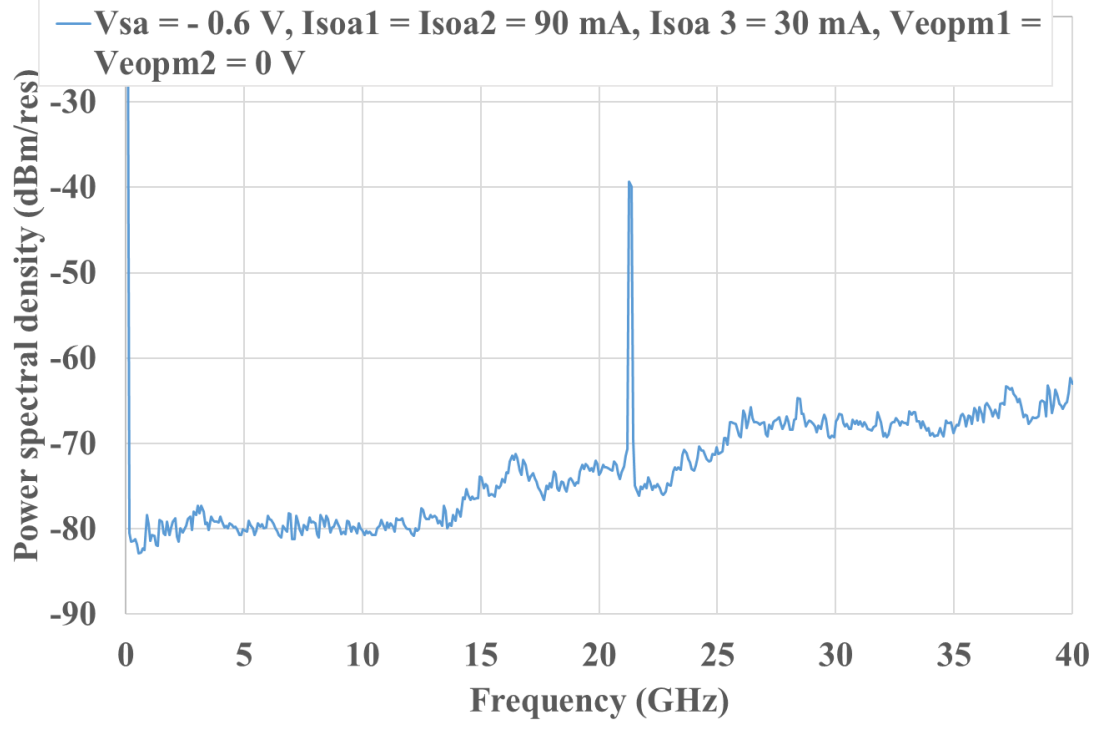

(a) 


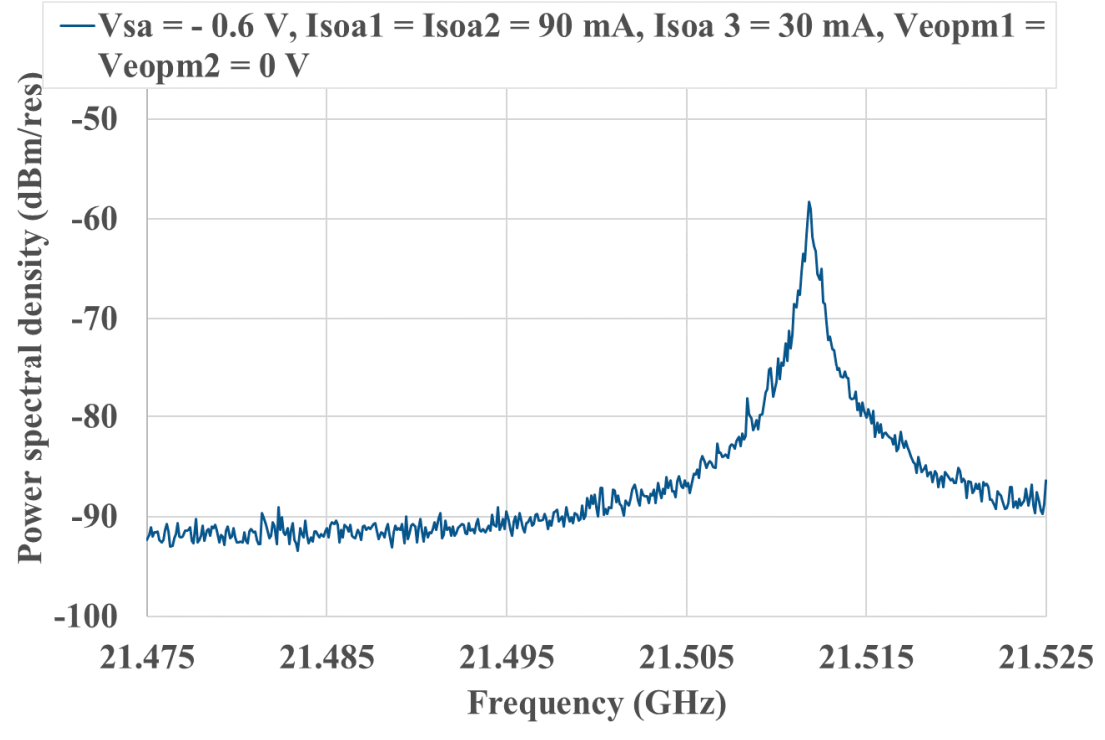

(b)

Figure 5. (a) RF spectrum when $\mathrm{Vsa}=-0.6 \mathrm{~V}$, Isoa1 $=\mathrm{Isoa} 2=90 \mathrm{~mA}$, Isoa3 $=30 \mathrm{~mA}$. Signal noise ratio is $35 \mathrm{~dB}$. (b) $\mathrm{RF}$ spectrum when $\mathrm{Vsa}=-0.6 \mathrm{~V}$, Isoa1 $=$ Isoa2 $=90 \mathrm{~mA}$, Isoa3 $=30 \mathrm{~mA}$. Linewidth is under $1 \mathrm{MHz}$.

\section{CONCLUSION}

We have presented a record broad band $(14 \mathrm{~nm} \sim 1.8 \mathrm{THz}$ at 3-dB level) optical frequency comb generated from a passively mode-locked quantum-well (QW) laser. This $21.5-\mathrm{GHz}$ colliding-pulse mode-locked laser with intracavity phase modulators was developed in an InP generic photonic integration technology platform. Under certain operating conditions, ultra-wide spectral bandwidth comb generation is demonstrated. However, the spectrum profile may be further optimized. The autocorrelation trace and RF spectrum have agreed with the mode locking nature, exhibiting a narrow pulse width of $0.35 \mathrm{ps}$ on a pedestal as well as an RF linewidth under $1 \mathrm{MHz}$ and 35-dB SNR.

\section{ACKNOWLEDGEMENT}

This project has received funding from the European Union's Horizon 2020 research and innovation programme under the Marie Sklodowska-Curie grant agreement No 642355 FiWiN5G and Spanish Ministerio de Economia y Competitividad DiDACTIC project TEC201347753-C3-3R.

\section{REFERENCES}

[1] P. J. Delfyett, S. Gee, M. T. Choi, H. Izadpanah, W. Lee, S. Ozharar, F. Quinlan, and T. Yilmaz, "Optical frequency combs from semiconductor lasers and applications in ultrawideband signal processing and communications," J. Lightw. Technol. 24(7), 2701-2719 (2006).

[2] U. Keller, "Recent developments in compact ultrafast lasers," Nature 424, pp. 831-838 (2003).

[3] A. R. Criado, P. Acedo, G. Carpintero, C. de Dios, and K. Yvind, "Observation of phase noise reduction in photonically synthesized sub-THz signals using a passively mode-locked laser diode and highly selective optical filtering," Opt. Exp. 20, 1253-1260 (2012).

[4] H. A. Haus, "Mode-locking of lasers," IEEE J. Sel. Top. Quantum Electron. 6(6), 1173-1185 (2000).

[5] J. Javaloyes, and S. Balle, "Mode-Locking in Semiconductor Fabry-Perrot Lasers," IEEE J. Quantum Electron. 46(7), 1023-1030 (2010).

[6] D. J. Derickson, R. J. Helkey, A. Mar, J. R. Karin, J. G. Wasserbauer, and J. E. Bowers, "Short pulse generation using multisegment mode-locked semiconductor lasers," IEEE J. Quantum Electron. 28(10), 2186-2202 (1992). 
[7] Y. K. Chen, M. C. Wu, "Monolithic colliding-pulse mode-locked quantum-well lasers," IEEE J. Quantum Electron. 28(10), 2176-2185 (1992).

[8] J. F. Martins-Filho, E. A. Avrutin, C. N. Ironside, and J. S. Roberts, "Monolithic multiple colliding modelocked quantum-well lasers: Experiment and theory," IEEE J. Sel. Top. Quantum Electron. 1(2), 539-551 (1995).

[9] T. Shimizu, I. Ogura, H. Yokoyama, "860 GHz rate asymmetric colliding pulse mode locked diode lasers," Electron. Lett. 33(22), 1868-1869 (1997).

[10]E. A. Avrutin, J. H. Marsh, E. L. Portnoi, "Monolithic and multi-GigaHertz mode-locked semiconductor lasers: Constructions experiments models and applications," Proc. IEE Optoelectron. 47, 251-278 (2000).

[11] M. Smit, X. Leijtens, E. Bente, J. Van der Tol, H. Ambrosius, D. Robbins, M. Wale, N. Grote, and M. Schell, "Generic foundry model for InP-based photonics," IET Optoelectron. 5(5), 187-194 (2011).

[12]R. G. M. P. Koumans, R. Van Roijen, "Theory for passive mode-locking in semiconductor laser structures including the effects of self-phase modulation dispersion and pulse collisions," IEEE J. Quantum Electron. 32(3), 478-492 (1996).

[13] K. A. Williams, M. G. Thompson, I. H. White, "Long wavelength monolithic mode-locked diode lasers," New J. Phys. 6, 179 (2004).

[14] M. S. Tahvili, Y. Barbarin, X. J. M. Leijtens, T. de Vries, E. Smalbrugge, J. Bolk, H. P. M. M. Ambrosius, M. K. Smit, and E. A. J. M. Bente, "Directional control of optical power in integrated InP/InGaAsP extended cavity mode-locked ring lasers," Opt. Lett. 36(13), 2462-2464 (2011).

[15]E. Bente, V. Moskalenko, S. Latkowski, S. Tahvili, L. Augustin and M. Smit, "Monolithically integrated InPbased modelocked ring laser systems," Proc. SPIE 9134, Semiconductor Lasers and Laser Dynamics VI 91340C (2014).

[16]C. Gordón, R. Guzmán, V. Corral, M.-C. Lo, and G. Carpintero, "On-Chip Multiple Colliding Pulse ModeLocked Semiconductor Laser," J. Lightw. Technol. 34, 4722-4728 (2016).

[17]M.-C. Lo, R. Guzmán, C. Gordón, and G. Carpintero, "Mode-locked laser with pulse interleavers in a monolithic photonic integrated circuit for millimeter wave and terahertz carrier generation," Opt. Lett. 42, 15321535 (2017).

[18] J. S. Parker, A. Bhardwaj, P. R. A. Binetti, Y.-J. Hung, and L. A. Coldren, "Monolithically integrated gainflattened ring mode-locked laser for comb-line generation," IEEE Photon. Technol. Lett. 24(2), 131-133 (2012).

[19] V. Moskalenko, J. Javaloyes, S. Balle, M. K. Smit, and E. A. J. M. Bente, "Theoretical Study of Colliding Pulse Passively Mode-Locked Semiconductor Ring Lasers with an Intracavity Mach-Zehnder Modulator,” IEEE J. Quantum Electron. 50(6), 415-422 (2014).

[20]V. Corral, R. Guzmán, C. Gordón, X. J. M. Leijtens, and G. Carpintero, "Optical frequency comb generator based on a monolithically integrated passive mode-locked ring laser with a Mach-Zehnder interferometer," Opt. Lett. 41(9), 1937-1940 (2016).

[21] M. G. Thompson, A. Rae, X. Mo, R. V. Penty, I. H. White, "InGaAs quantum-dot mode-locked laser diodes," IEEE J. Sel. Top. Quantum Electron. 15(3), 661-672 (2009).

[22] G.-H. Duan, A. Shen, A. Akrout, F. V. Dijk, F. Lelarge, F. Pommereau, O. LeGouezigou, J.-G. Provost, H. Gariah, F. Blache, F. Mallecot, K. Merghem, A. Martinez, A. Ramdane, "High performance InP-based quantum dash semiconductor mode-locked lasers for optical communications," Bell Labs Tech. J. 14(3), 63-84 (2009).

[23]R. Rosales, K. Merghem, A. Martinez, A. Akrout, J.-P. Tourrenc, A. Accard, F. Lelarge, and A. Ramdane, "InAs/InP quantum-dot passively mode-locked lasers for 1.55- $\mu \mathrm{m}$ applications," IEEE J. Sel. Top. Quantum Electron. 17(6), 1698-1714 (2011).

[24] R. Rosales, S. G. Murdoch, R.T. Watts, K. Merghem, A. Martinez, F. Lelarge, A. Accard, L. P. Barry, and A. Ramdane, "High performance mode locking characteristics of single section quantum dash lasers," Opt. Exp. 20(8), 8649-8657 (2012).

[25] V. Moskalenko, S. Latkowski, S. Tahvili, T. de Vries, M. Smit, and E. Bente, "Record bandwidth and subpicosecond pulses from a monolithically integrated mode-locked quantum well ring laser," Opt. Exp. 22(23), 28865-28874 (2014).

[26] V. Moskalenko, J. Koelemeij, K. Williams, and E. Bente, "Study of extra wide coherent optical combs generated by a QW-based integrated passively mode-locked ring laser," Opt. Lett. 42(7), 1428-1431 (2017).

[27] SMART Photonics B. V., http://www.smartphotonics.nl/

[28] Joint European Platform for Photonic Integration of Components and Circuits, http://www.jeppix.eu/ 
[29] C. Gordón, R. Guzmán, V. Corral, X. Leijtens, and G. Carpintero, "On-chip colliding pulse mode-locked laser diode (OCCP-MLLD) using multimode interference reflectors," Opt. Exp. 23(11), 14666-14676 (2015).

[30] I. N. Duling, [Compact Sources of Ultrashort Pulses], Cambridge, U.K.: Cambridge Univ., (1995).

[31] R. Trebino, [Frequency-Resolved Optical Gating: The Measurement of Ultrashort Laser Pulses], Boston, MA, USA: Kluwer, (2002). 